\title{
Murrayanine suppresses the proliferation and metastasis of human breast cancer cells via induction of apoptosis and inhibition of RANK/RANKL signaling pathway
}

\author{
Jingnan zhang ${ }^{1}$, Feng Wang ${ }^{2}$, Gengbao Qu² and Baokai Wang ${ }^{2 *}$
}

\begin{abstract}
Murrayanine and its derivatives have been shown to exhibit anticancer activities against different types of human cancer cells. However, the effects of murrayanine on the proliferation and metastasis of breast cancer cells are yet to be studied. The present study was designed to evaluate the effects of murrayanine on the proliferation and metastasis of human breast cancer via regulation of RANK/RANKL pathway. The results showed RANK/RANKL pathway to be highly activated in human breast cancer tissues and cell lines. However, treatment of the CAMA-1 breast cancer cells with murrayanine $(0,9,18$ and $36 \mu \mathrm{M})$ caused a significant $(P<0.05)$ decline in the expression of RANK, RANKL and OPG in CAMA-1 cells. Additionally, murrayanine inhibited the growth of CAMA-1 cells with an $I_{50}$ of $18 \mu \mathrm{M}$. The antiproliferative of murrayanine were found be due to its ability to inhibit the expression of RANK, RANKL and OPG in CAMA-1 cells. To unveil if murrayanine exerted its effects via inhibition of RANK/RANKL pathway, the expression of RNAK was knocked down in CAMA-1 cells. It was found that murrayanine and RANK silencing both inhibited the growth CAMA-1 cells via induction of apoptosis. Additionally, murrayanine and RANK silencing both inhibited the migration, invasion and epithelial to mesenchymal transition of the CAMA-1 cells. Taken together, murrayanine exhibits significant anticancer activity against the breast cancer cells via induction of apoptosis and inhibition of RANK/RANKL signaling pathway. These findings suggest that murrayanine may prove to be a beneficial lead molecule for the development of breast cancer chemotherapy.
\end{abstract}

Keywords: Breast cancer, Murrayanine, RANK/RANKL pathway, Apoptosis, Metastasis, Anticancer

\section{Introduction}

Breast cancer is one of the most frequent gynecological disorders which can be understood by the fact that more than 1.5 million females are diagnosed with this malignancy each year throughout the globe which sums up to nearly the $25 \%$ of total annually reported cancer cases in women [1,2]. Numerous risk factors have been shown to be linked with breast cancer like age, genetic

\footnotetext{
*Correspondence: baokaiwang33@gmail.com

2 Department of Breast Surgery, Fengtai District, Beijing Tiantan Hospital, Capital Medical University, No.119 South 4th Ring West Road, Beijing 100070, China

Full list of author information is available at the end of the article
}

mutations, family history etc. with growing age being the most dominant risk factor in developing countries [3]. Breast cancer is considered as the second deadliest cancer of the women and resulted in 570,000 deaths in 2015 [4]. Fortunately, near about $90 \%$ of breast cancer patients present the non-metastatic disease state and show higher survival chances. However, the metastatic breast cancer despite application of advanced therapeutic procedures is seen with extremely high chances of disease recurrence and thus remains nearly incurable till date. Therefore, the scientific investigations are continuously aimed at identifying more effective treatment measures against breast cancer and the metabolites derived from biological 
sources are preferably being studied for their effectiveness against the said disease. Numbers of natural molecules containing the carbazole nucleus have been stated for their anticancer potential and cytotoxic effects against different types of human cancer cell lines including the breast cancer cells, many of which have been reported for their therapeutic applicability even in the pre-clinical trials [5].

Murrayanine is a carbazole type of plant-based alkaloid which is isolated from the bark of the stem of Murraya koenigii Spreng [6]. It has been given as a treatment for human cancer in traditional Chinese system [7]. Recent studies have shown that murrayanine and its derivatives exhibit considerable anti-proliferative effects against the human cancer cells [8-10]. Zhou et al., showed that murrayanine suppresses the proliferation of the human oral cancer cells by blocking the AKT/mTOR and Raf/MEK/ ERK signalling pathways [8]. Similarly, some murrayanine-Schiff's base derivatives have been shown to exhibit potent anticancer effects $[9,10]$. In yet another study Zhang et al., showed that murrayanine triggers cell cycle arrest of the human lung cancer cells via inhibition of phosphorylated p38 expression [11]. However, the anticancer effects of murrayanine have not been evaluated against the human breast cancer. Thus, the current study represents elucidation of the same together with exploration of the underlying mechanism of action. Murrayanine targeted the RANK/RANKL signaling pathway which was shown to be highly active in breast cancer tissues and cells. Apoptosis was induced in breast cancer cells treated with murrayanine and the cancer cells exhibited significantly lower growth and metastatic potential. The decline in breast cancer cell growth by murrayanine resembled the effects of transcriptional silencing of RANK. The present study established the anticancer activity of murrayanine against breast cancer cells via the inhibition of RANK/RANKL signaling pathway.

\section{Materials and methods}

\section{Clinical specimens}

A total of 20 clinical specimens corresponding to breast cancer and normal-adjacent tissues were obtained from the breast cancer patients who underwent surgery at the Department of Breast Surgery, Beijing Tiantan Hospital, Capital Medical University, Beijing, 100,070, China. None of the patients had received chemotherapy at the time of specimen collection. Every patient was informed in advance and tissues were collected only after proper consent and written undertaking. The study on human tissues was performed strictly under the ethical guidelines of our institutional committee. The specimens were immediately frozen after collection using liquid nitrogen and stored at $-80{ }^{\circ} \mathrm{C}$ till future use.

\section{Maintenance of cell lines and cell transfection}

After being purchased from American Type Culture Collection (ATCC, USA), SK-BR-3 and CAMA-1 breast cancer cell lines and normal breast epithelial cell line, MB-157 were maintained in humid atmosphere of $5 \% \mathrm{CO}_{2} / 95 \%$ air at $37{ }^{\circ} \mathrm{C}$ in a humidified $\mathrm{CO}_{2}$ incubator. The Dulbecco's Modified Eagle Medium (DMEM, Sigma-Aldrich) containing 10\% fetal bovine serum (FBS, Thermo Fisher Scientific) was used for in vitro culturing of all the three cell lines. CAMA-1 cancer cell line was transfected with RNAi construct of RANK gene (siRANK, RiboBio) using Lipofectamine 2000 following the manufacturer guidelines. The si-NC RNA transfected cells were used as the negative control.

\section{qRT-PCR and western blotting}

Using TriZOL reagent (Thermo Fisher Scientific), the total RNA was extracted from tissues (cancer and normal) as well as from the cell lines as per the standard procedure. With the help of PrimeScript RT reagent kit (Takara Bio), $2 \mu \mathrm{g}$ of total RNA was used to synthesize the complementary DNA (cDNA). SYBR ${ }^{\mathrm{TM}}$ Green PCR Master Mix (Thermo Fisher Scientific) was used for performing qRT-PCR on QuantStudio 3.0 PCR system (Applied Biosystems) for determining the relative expression of RANK, RANKL and OPG genes. The expression analyses were normalized to the GAPDH. The quantitative analysis was performed with the help of $2^{-\Delta \Delta \mathrm{Ct}}$ method. The primers used in the study are listed in Table 1.

\section{Western blotting}

Western blotting was used for protein expression analysis. Briefly, total proteins were extracted from tissues and cells through their digestion with RIPA lysis and extraction buffer (Thermo Fisher Scientific) supplemented with $10 \mathrm{mM}$ PMSF (Beyotine). The protein lysates were resolved on $8-10 \%$ SDS-polyacrylamide gels and electrophoretically transferred to PVDF membranes

Table 1 List of the primer sequences used for qRT-PCR gene expression analyses in the present study

\begin{tabular}{lll}
\hline Primer & Direction & Sequence \\
\hline RANK & Forward & 5'-AGCATTATGAGCATCTGG-3' \\
& Reverse & 5'-CAGCAAGCATTTATCTTC-3' $^{\prime}$ \\
RANKL & Forward & $5^{\prime}$-GACAGGCACGGACTCGTA-3' \\
& Reverse & $5^{\prime}$-GCTCATGCTAGTCGTCTA-3' \\
OPG & Forward & $5^{\prime}$-AGCATCACTACGTAGGAC-3' \\
& Reverse & $5^{\prime}$-ACGTCATGCGATCACATA-3' \\
GADPH & Forward & $5^{\prime}$-AAG CCTGCCGGTGACTAA C -3' \\
& Reverse & $5^{\prime}$-GCATCACCCGGAGGAGAA AT -3' \\
\hline
\end{tabular}


(Millipore). The membranes were blocked using 5\% skimmed milk and then incubated overnight with corresponding primary antibodies (Cell Signaling Technologies) at $4{ }^{\circ} \mathrm{C}$. Following, TBS/Tween-20 was used to wash the PVDF membranes which were re-incubated with horseradish peroxidase-conjugated secondary antibodies for $2 \mathrm{~h}$ at room temperature. Final, the protein blots were developed using an ECL detection kit (Millipore) and the $\beta$-actin protein was used for normalizing the protein expressions.

\section{Cell viability assay}

The effects of murrayanine, administered in varying doses form $0 \mu \mathrm{M}$ to $160 \mu \mathrm{M}$ for $24 \mathrm{~h}$, on the viability of CAMA-1 cancer cells and MB-157 normal cells were analyzed using 3-(4,5-dimethylthiazol-2yl-yl)-2,5-diphenyl tetrazolium bromide (MTT) assay kit (Chemicon, Temecula) as per the manufacturer guidelines.. Briefly, $2 \times 10^{3}$ cells were seeded per well of 96-well plates (Falcon, Becton Dickinson Labware) and incubated at $37{ }^{\circ} \mathrm{C}$ with murrayanine $(0,2.5,5,10,20,40,80$ or $160 \mu \mathrm{M})$. After murrayanine administration, the relative percentage of viable cells was determined using MTT assay as described previously by recording $\mathrm{OD}_{570}$ using spectrophotometer [12]. MTT assay was also used for analyzing the proliferation of murrayanine treated $(18 \mu \mathrm{M})$ and siRANK transfected CAMA-1 cancer cells with reference to corresponding negative control cells.

\section{EdU assay}

EdU staining assay was performed to estimate the proliferative viability of murrayanine $(18 \mu \mathrm{M})$ treated and si-RANK transfected CAMA-1 cells along with the respective control cells. In brief, the cancer cells after being treated with murrayanine and those transfected with si-RANK for $24 \mathrm{~h}$ together with respective controls were collected, fixed with $4 \%$ paraformaldehyde for $20 \mathrm{~min}$ at room temperature. The cells were then incubated with EdU ( $20 \mathrm{mmol} / \mathrm{L})$ for $1.5 \mathrm{~h}$ at room temperature at which the cells were analyzed using fluorescent microscope for examining the relative percentage of EdU positive cells. DAPI was used as background stain.

\section{Annexin V/PI staining assays}

The analysis of apoptosis of murrayanine $(18 \mu \mathrm{M})$ treated and si-RANK transfected CAMA- 1 cells for $24 \mathrm{~h}$ at $37^{\circ} \mathrm{C}$ in DMEM with 10\% FBS in 6-well plates. Centrifugation was performed for harvesting the cells which were washed with cold PBS thrice. The cells were then resuspended in binding buffer and mixed with $10 \mu \mathrm{L}$ Annexin V-FITC plus $10 \mu \mathrm{L}$ propidium iodide (Beyotime) at $4{ }^{\circ} \mathrm{C}$ in dark for $25 \mathrm{~min}$. The annexin V-FITC/PI dual stained cells again washed with PBS three times and then resuspended in $250 \mu \mathrm{L}$ binding buffer. Flow cytometry (BD, FACS Calibur) was then performed for determining the relative percentage of apoptosis.

\section{Wound-healing assays}

A wound-healing assay was used for the determination of migration of breast cancer cells administered with $18 \mu \mathrm{M}$ murrayanine or transfected with si-RANK with reference to respective controls. In brief, $2 \times 10^{5}$ cancer cells were plated in 6-well plates and incubated for $24 \mathrm{~h}$ at $37^{\circ} \mathrm{C}$. A sterile $200 \mu \mathrm{l}$ pipette tip was then used for creating an artificial wound on the cell surface. The wound area was cleared off the suspended cells by PBS washing, the wound was photographed under light microscope and then the plates were incubated again for $24 \mathrm{~h}$ at $37^{\circ} \mathrm{C}$. The wounds were again photographed at $24 \mathrm{~h}$ and the wound-width between the lines was measured using Image J software.

\section{Transwell assay}

In vitro invasion of murrayanine treated or si-RANK transfected cancer cells with reference to corresponding negative control cells was investigated with the help of a 24-well insert transwell Matrigel invasion assay $(8.0 \mu \mathrm{m}$, Corning). Approximately, $2 \times 10^{5}$ cells suspended in 200 $\mu \mathrm{L}$ of serum-free DMEM plated into upper chamber of transwell pre-coated with $1 \mu \mathrm{g} / \mu \mathrm{L}$ Matrigel (BD Biosciences).The bottom wells received complete medium with $10 \%$ FBS for stimulating the cell invasion. After $24 \mathrm{~h}$ incubation at $37^{\circ} \mathrm{C}$, the cells unable to penetrate the membrane were swabbed off using a cotton swab while those adhering to the membrane's lower surface were PBS washed, ethanol fixed, stained with a $0.1 \%$ crystal violet solution and visualized and manually counted using light microscopy.

\section{Statistical analysis}

The results are shown as mean \pm SD calculated using three replicates for each experiment. All the statistical analyses were performed using SPSS software. Students $\mathrm{t}$-test was used for assessing the statistical differences and the values of $\mathrm{P}<0.05$ were taken as representative of statistically significant difference.

\section{Results \\ RANK/RANKL signaling pathway is over-expressed} in breast cancer

The western blot analyses of RANK, RANKL and OPG proteins were performed from breast cancer and normal adjacent tissues. The cancer tissues were shown to express relatively higher expression of all these proteins (Fig. 1a). The qRT-PCR based expression analysis of transcript levels of RANK, RANKL and OPG genes was 

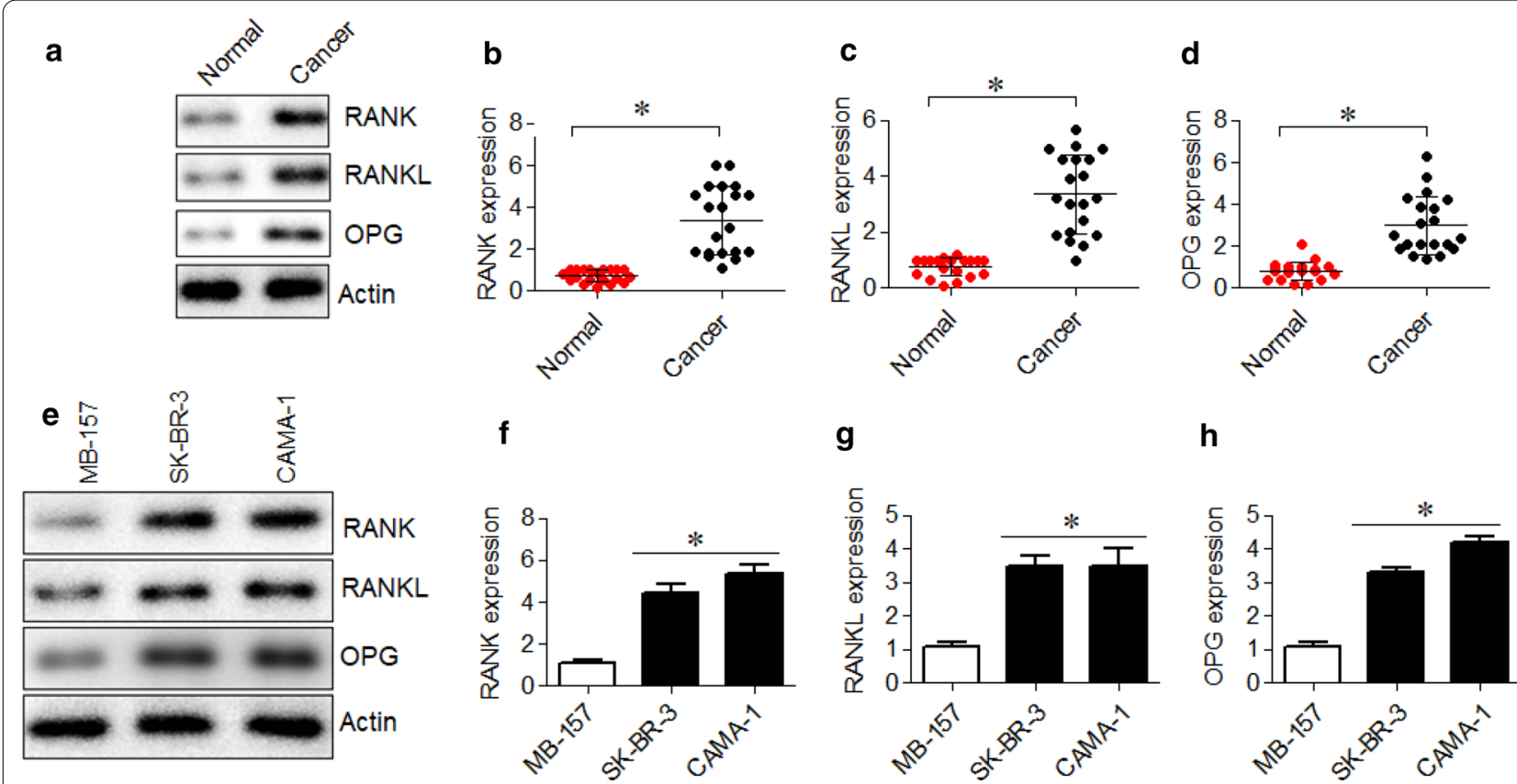

Fig. 1 Activation of RANK/RANKL pathway in breast cancer. a Western blots showing expression of RANK, RANKL and OPG proteins in breast cancer and normal adjacent tissues. The qRT-PCR analysis of $\mathbf{b}$ RANK $\mathbf{c}$ RANKL and $\mathbf{d}$ OPG transcripts in breast cancer and normal adjacent tissues $\mathbf{e}$ Western blots showing RANK, RANKL and OPG expression in breast cancer and normal breast epithelial cell line. The qRT-PCR analysis of $\mathbf{f}$ RANK $\mathbf{g}$ RANKL and $\mathbf{h}$ OPG transcript levels from breast cancer and normal breast epithelial cell line. Experiments were performed using three replicates and values are presented as mean $\pm S D\left({ }^{*} P<0.05\right)$

also in coherence with the western blotting results. The breast cancer tissues were shown to exhibit significantly $(\mathrm{P}<0.05)$ higher transcript levels of RNAK, RANKL and OPG genes (Fig. 1b-d). The RANK, RANKL and OPG protein and gene expression levels were also determined from two breast cancer cell lines (SK-BR-3 and CAMA-1) and a normal breast epithelial cell line, MB-157. Results showed that both SK-BR-3 and CAMA-1 cancer cells expressed significantly $(\mathrm{P}<0.05)$ higher protein and transcript levels of RANK, RANKL and OPG (Fig. 1e-h). The results thus suggest that breast cancer exhibits overexpression of RANK/RANKL signaling pathway. CAMA-1 cell line was used for further experimentation.

\section{Murrayanine targeted RANK/RANKL pathway in breast cancer cells}

To decipher the effects of murrayanine treatment on MB-157 normal cells and on CAMA-1 cancer cells, both cell lines were treated with murrayanine $(0,2.5,5,10,20$, 40,80 or $160 \mu \mathrm{M}$ ) for $24 \mathrm{~h}$ and their viability was subsequently assessed using MTT assay. The MB-157 normal cells exhibited dose dependent viability loss by murrayanine with an $\mathrm{IC}_{50}$ of $140 \mu \mathrm{M}$ (Fig. 2a). Similarly, the CAMA-1 cancer cell viability declined with increasing doses of murrayanine, but the effects were more severe as suggested by $\mathrm{IC}_{50}$ value of $18 \mu \mathrm{M}$ (Fig. 2b). The expression analysis of RANK, RANKL and OPG proteins was performed form CAMA-1 cancer cells administered with 0 , 9,18 or $36 \mu \mathrm{M}$ of murrayanine. The results showed that the expression levels of RANK, RANKL and OPG proteins significantly $(\mathrm{P}<0.05)$ declined under murrayanine treatments and the expressional repression showed dose dependence (Fig. 2c-f). This indicates that murrayanine targeted RANK/RANKL pathway in breast cancer cells to inhibit their growth.

\section{Murrayanine inhibited breast cancer cell growth via RANK repression driven apoptosis}

To examine whether the murrayanine inhibited the in vitro growth of CAMA-1 through RANK repression, the CAMA-1 cancer cells were treated with $18 \mu \mathrm{M}$ murrayanine and MTT assay was performed to analyze their proliferation together with the RANK downregulating CAMA-1 cells. The cell proliferations were compared with corresponding negative controls at $0 \mathrm{~h}$, $12 \mathrm{~h}, 24 \mathrm{~h}, 36 \mathrm{~h}$ and $48 \mathrm{~h}$. The proliferation rates of CAMA-1 cancer cells treated with murrayanine were significantly $(\mathrm{P}<0.05)$ lower than that of control cells at every time point except $0 \mathrm{~h}$ (Fig. 3a). The RANK gene silencing also exerted similar effects on the proliferation of the CAMA-1 cancer cells and the siRANK transfected cells exhibited significantly higher 


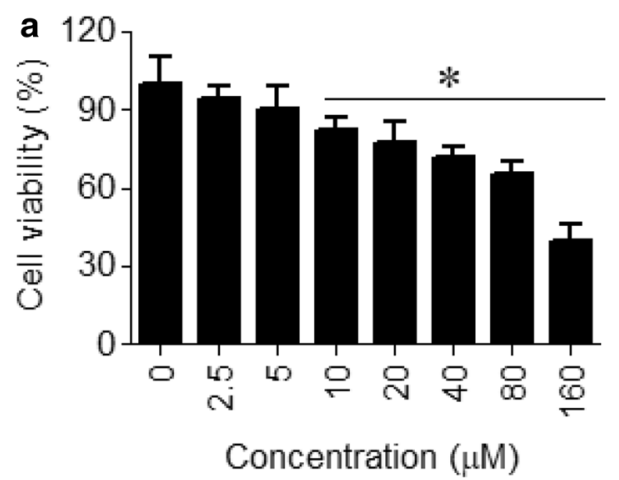

c
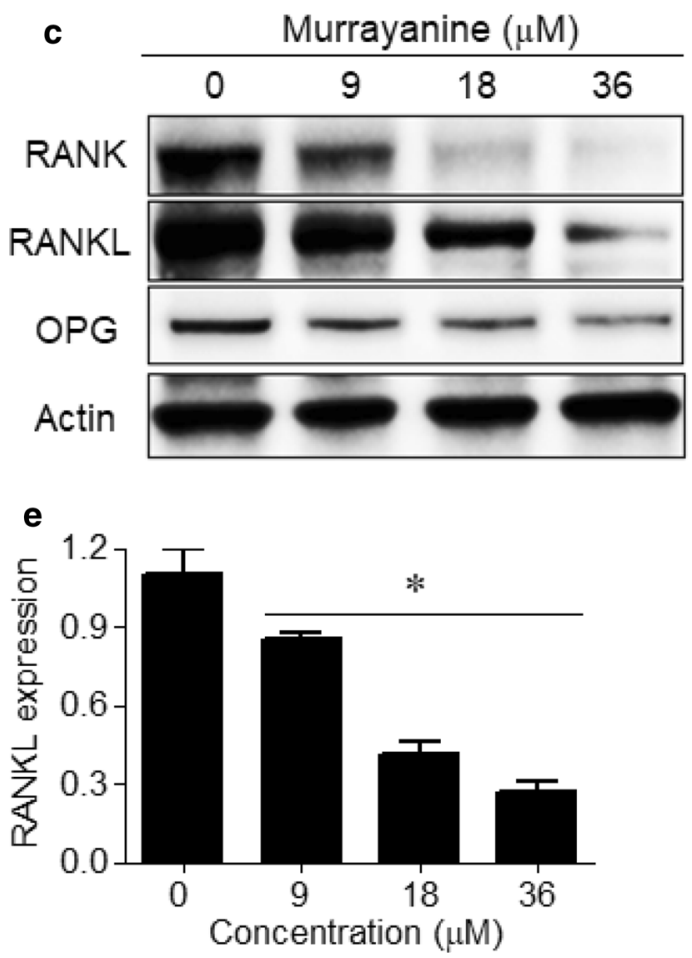

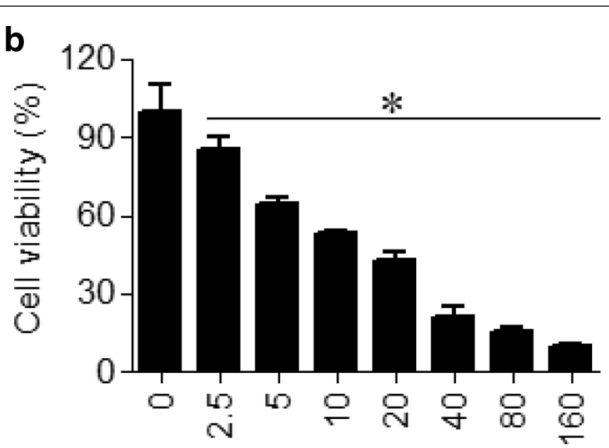

Concentration $(\mu \mathrm{M})$

d
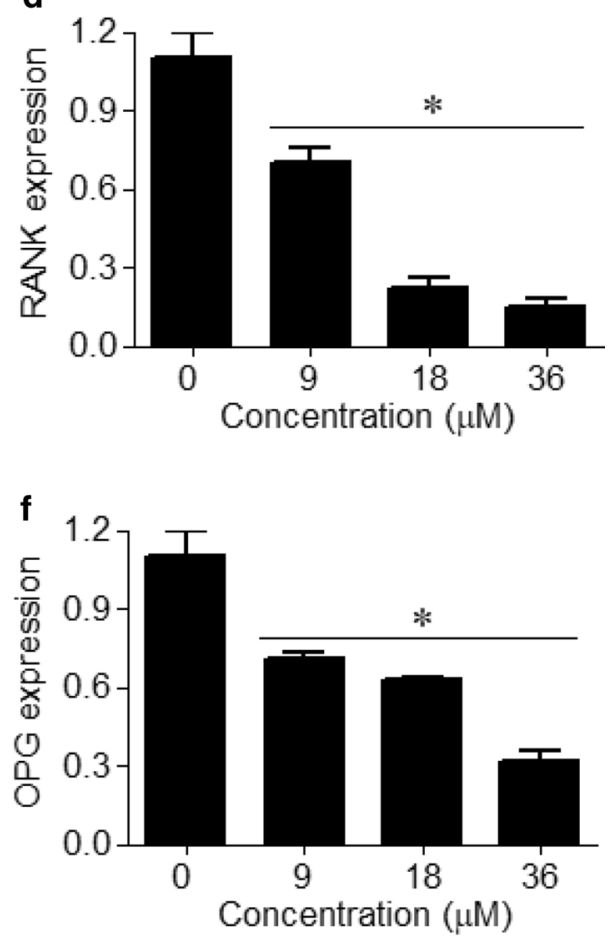

Fig. 2 Inhibition of breast cancer cell proliferation by murrayanine via RANK/RANKL signaling pathway. Viability of a MB-157 normal breast epithelial cells and $\mathbf{b}$ CAMA-1 breast cancer cells treated with indicated concentrations of murrayanine as determined by MTT assay. $\mathbf{c}$ Western blotting of RANK, RANKL and OPG proteins from CAMA-1 cancer cells treated with 0, 9, 18 or $36 \mu \mathrm{M}$ murrayanine. Relative expression of $\mathbf{d}$ RANK $\mathbf{e}$ RANKL and $\mathbf{f} O P G$ in CAMA-1 cancer cells treated with $0,9,18$ or $36 \mu \mathrm{M}$ murrayanine. Experiments were performed using three replicates and values are presented as mean $\pm \mathrm{SD}\left({ }^{*} \mathrm{P}<0.05\right)$

proliferation in comparison to the respective cancer cells (Fig. 3b). EdU assay was used for analyzing the proliferative viability of CAMA-1 cancer cells administered with $18 \mu \mathrm{M}$ murrayanine and those transfected with si-RANK with respect to the corresponding negative control cancer cells. The relative percentage of EdU positive cells was significantly $(\mathrm{P}<0.05)$ lower under murrayanine treatment and si-RANK transfection as compared to the negative control cells (Fig. 3c, d). Annexin V-FITC/PI staining was used for the study of apoptosis of CAMA-1 cancer cells administered with $18 \mu \mathrm{M}$ murrayanine and those transfected with si-RANK with respect to the corresponding negative control cancer cells. The results indicated that the percentage of both murrayanine treated and siRANK transfected MCF- 7 apoptotic cancer cells was significantly higher than the corresponding negative control cells (Fig. 3e). Additionally, the expression of Bax and cleaved caspase- 3 was increased and that of $\mathrm{Bcl}-2$ and $\mathrm{Bcl}-\mathrm{xl}$ was decrease under both murrayanine 
a
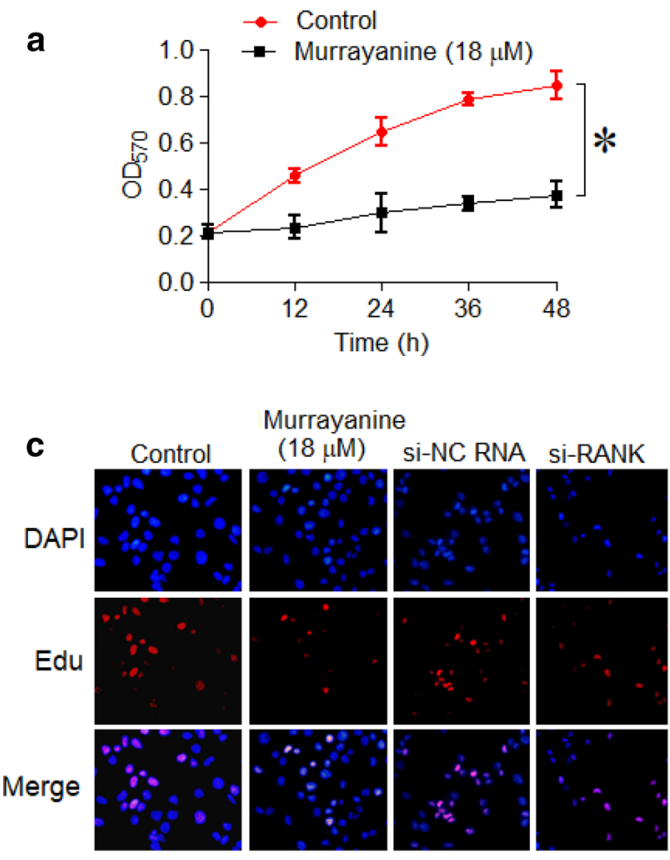

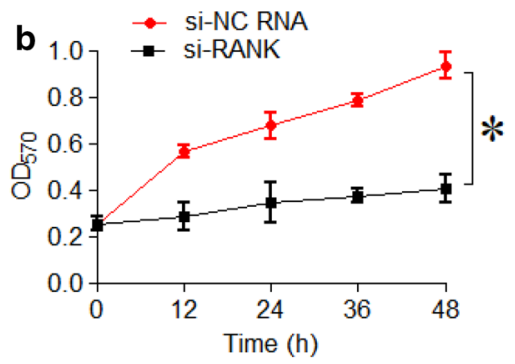

d
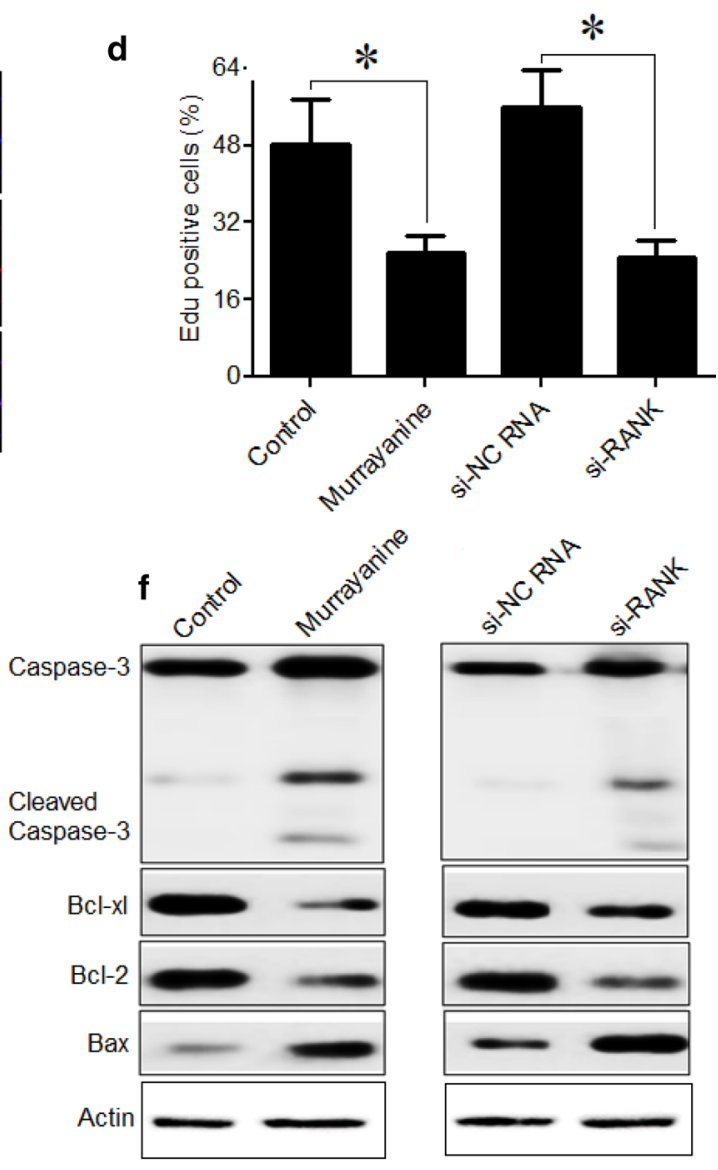

e
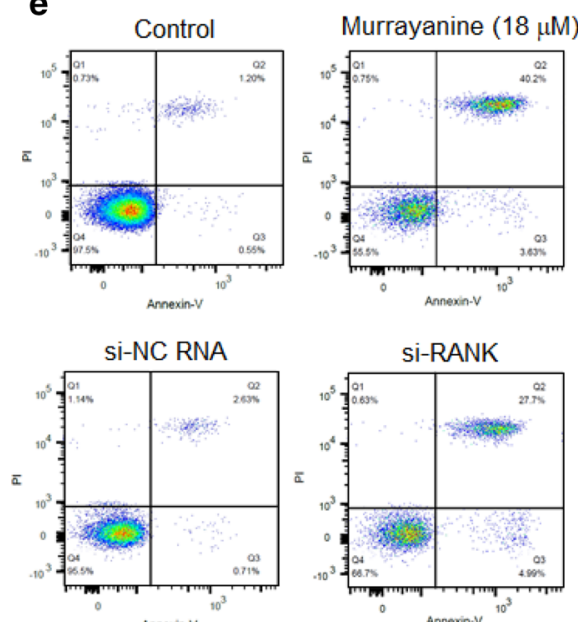

Fig. 3 Induction of apoptosis in breast cancer cells by murrayanine via RANK/RANKL signaling pathway. a Viability of untreated control and murrayanine treated $(18 \mu \mathrm{M})$ CAMA-1 cancer cells at 0, 12, 24, 36 or $48 \mathrm{~h}$. b Viability of si-NC RNA and si-RANK transfected CAMA-1 cancer cells at 0, $12,24,36$ or $48 \mathrm{~h}$ as determined by MTT assay. $\mathbf{c}$ EdU staining analysis showing proliferation of untreated, murrayanine treated (18 $\mu \mathrm{M})$ si-NC RNA, and si-RANK transfected CAMA-1 cancer cells at $24 \mathrm{~h}$ culture duration. $\mathbf{d}$ Relative percentage of EdU positive in murrayanine treated (18 $\mu \mathrm{M})$ si-NC RNA, and si-RANK transfected CAMA-1 e Annexin V/PI assay showing percent apoptosis of murrayanine treated (18 $\mu$ M) si-NC RNA, and si-RANK transfected CAMA-1 cells $\mathbf{f}$ Western blots showing the expression of Caspase-3, BCl-xl, BCl-2 and Bax in murrayanine treated (18 $\mu \mathrm{M})$ si-NC RNA, and si-RANK transfected CAMA-1 cells. Experiments were performed using three replicates and values are presented as mean $\pm S D\left({ }^{*} P<0.05\right)$

treatment or RANK silencing (Fig. 3f) The results thus indicate that murrayanine inhibits the proliferation of breast cancer cells by inducing apoptosis and the latter might be triggered via RANK gene silencing.

\section{Murrayanine and RANK silencing reduced metastatic potential of breast cancer cells}

To investigate the effects of murrayanine on the metastatic potential of breast cancer cells and whether the 
same was exerted through RANK gene down-regulation, firstly the migration of both murrayanine $(18 \mu \mathrm{M})$ and RANK repressing CAMA-1 cells was studied using wound-healing assay and compared with that of the corresponding negative control CAMA-1 cancer cells. The results showed that under both murrayanine administration and RANK gene silencing, the migration of CAMA-1 cancer cells was significantly $(\mathrm{P}<0.05)$ reduced (Fig. 4a). The analysis of in vitro invasion was made through transwell assay and the results were similar to those of the wound-healing assay. Both murrayanine and si-RANK transfected CAMA-1 breast cancer cells exhibited significantly lower invasiveness as compared to the negative control cells (Fig. 4b). Moreover, the inhibition and migration and invasion of CAMA-1 cells was also associated with the inhibition of the MMP-2 and MMP-9 expression (Fig. 4c). Whether murrayanine modulates the EMT of breast cancer cells, the murrayanine treated breast cancer cells along with those transfected for RANK gene silencing were examined for the protein expression levels of EMT molecular markers. The expression of epithelial marker protein (E-cadherin) significantly increased while those of mesenchymal markers (Vimentin and N-cadherin) significantly decreased both

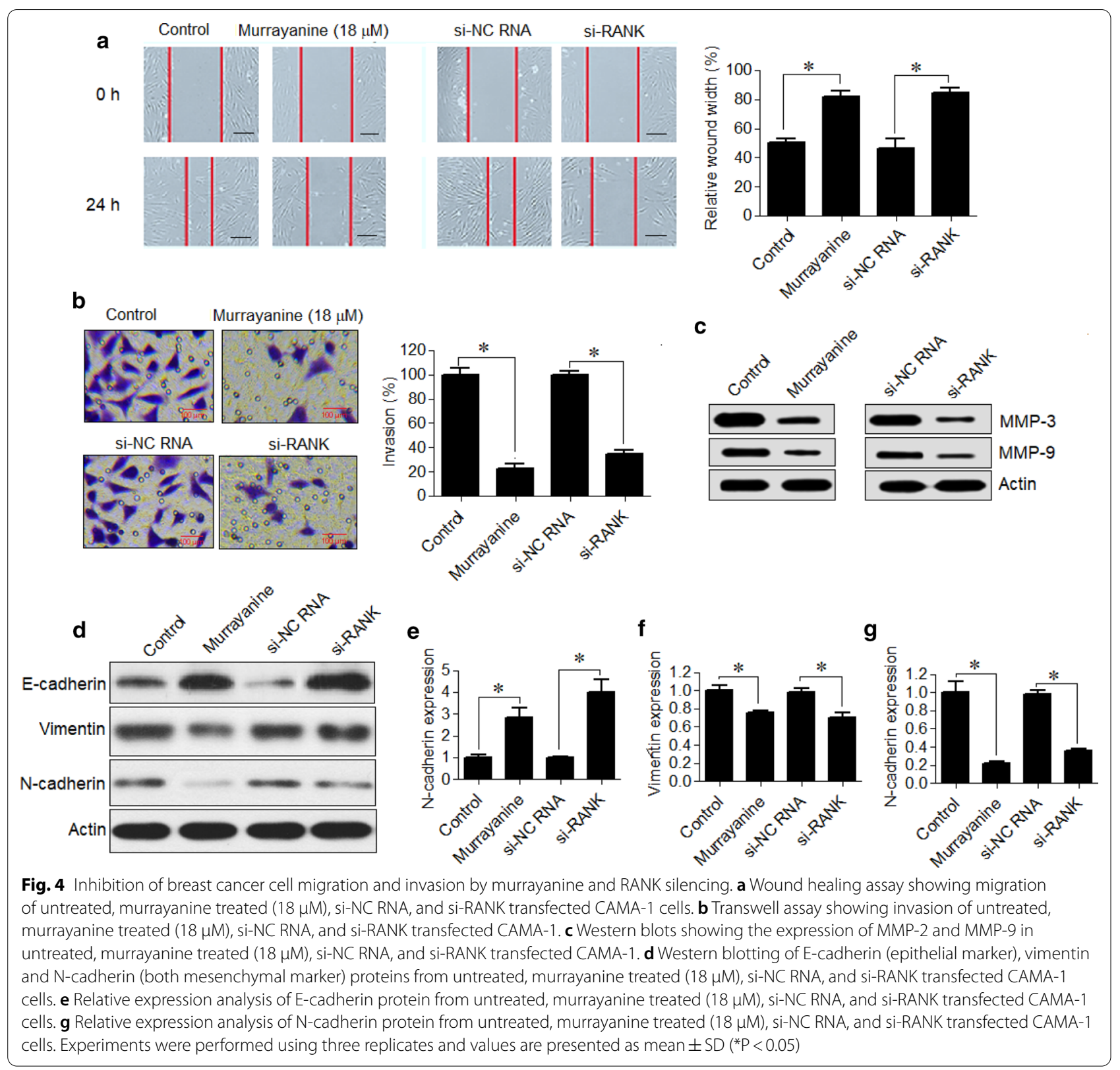


under murrayanine administration and si-RANK transfection (Fig. $4 \mathrm{~d}-\mathrm{g}$ ). The results thus indicate that murrayanine inhibits the metastasis of breast cancer cells and the same might be resulting from its potential to repress RANK protein and hence the RANK/RANKL signaling cascade in breast cancer cells.

\section{Discussion}

At present, the widely exercised treatment procedure against different cancer types includes the chemotherapy wherein a drug molecule either singly or in combination with other chemical compounds, is targeted to prevent the carcinogenic adversities [13]. These drug molecules have enormous cytotoxic potential against the human cancer cells but at the same time the procedure is seen with a limitation to execute adverse effects on the normal body cells also [14]. To address this drawback, the researchers are in continuous hunt for the molecules with very fewer side effects to replace the present day chemotherapeutic drug moieties. The preclinical studies have revealed that natural compounds might aid in chemotherapy by enhancing the cytotoxic effects of the chemotherapeutic drug molecules or by minimizing the drug resistance of cancer cells [15]. Moreover, the natural occurring metabolites like carbazole alkaloids have been reported to exhibit pro-apoptotic and anti-proliferative effects against several types of human cancer cells through multiple signaling cascades $[16,17]$. In the present study, a plant based carbazole alkaloid murrayanine was shown to target a highly active RANK/RANKL signaling pathway in breast cancer cells. The enrichment of RANK cell surface glycoprotein (receptor activator nuclear factor) together with over-expression of its ligand, RANKL (receptor activator nuclear factor- $\mathrm{kB}$ ligand) frequently in the tumor-microenvironment, which together are coordinated by osteoprotegrin (OPG) receptor protein, has been deduced to possess significant functional implication in tumor growth and development [18]. The breast cancer tissues, and cell lines exhibited over-expression of RANK, RANKL as well as that of OPG protein. The administration of murrayanine led to significant decline in the expression of RANK/RANKL signaling pathway and thus signifies its clinical significance [19]. The repression of RANK has been proven to induce apoptosis in cancer cells [20]. The induction of apoptosis in breast cancer cells by murrayanine might thus be mediated through this mechanism. Murrayanine has been previously shown to induce apoptosis in oral cancer cells also [8]. In the current study, murrayanine was besides shown to reduce the metastatic potential of breast cancer cells by inhibiting their migration, invasion and epithelial to mesenchymal transition. Similar results were confirmed for the transcriptional silencing of
RANK which indicates that murrayanine targets RANK/ RANKL pathway to inhibit breast cancer metastasis [21]. Collectively, the current study depicted that RANK/ RANKL signaling pathway is highly active in breast cancer to promote its growth and metastasis. Murrayanine induces apoptosis in human breast cancer cells via inhibition of the RANK/RANKL signaling pathway. Moreover, murrayanine could also suppress the migration and invasion of the breast cancer cells. Collectively, these findings point towards the effectiveness of murrayanine to act as a lead therapeutic molecule against breast cancer in future.

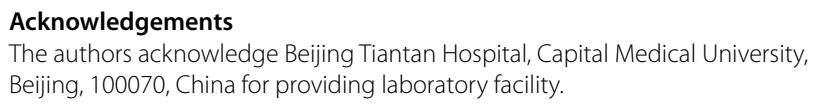

\section{Authors' contributions}

JZ and BW conceived the idea and designed the study. JZ, FW, GQ and BW performed the experiments and collect the data. JZ, FW and GQ were involved in the statistical analysis. BW supervised the work and drafted the manuscript. All authors read and approved the final manuscript.

\section{Funding}

Not applicable.

\section{Availability of data and materials}

Not applicable.

Declarations

Ethics approval and consent to participate

The study was approved by the research ethics committee of Beijing Tiantan Hospital, Capital Medical University, Beijing, 100070, China under approval number CMU-789HT-2019.

\section{Consent for publication}

Not applicable.

\section{Author details}

1 Department of Pharmacy, Linyi City Central Hospital, Yishui 276400

Shandong, China. ${ }^{2}$ Department of Breast Surgery, Fengtai District, Beijing Tiantan Hospital, Capital Medical University, No.119 South 4th Ring West Road, Beijing 100070, China.

Received: 4 November 2020 Accepted: 24 February 2021

Published online: 16 March 2021

\section{References}

1. DeSantis CE, Ma J, Goding SA, Newman LA, Jemal A (2017) Breast cancer statistics, 2017, racial disparity in mortality by state. Cancer J Clin. 67(6):439-448

2. Torre LA, Islami F, Siegel RL, Ward EM, Jemal A. Global cancer in women: burden and trends.

3. Majeed W, Aslam B, Javed I, Khaliq T, Muhammad F, Ali A, Raza A (2014) Breast cancer: major risk factors and recent developments in treatment. Asian Pac J Cancer Prev 15(8):3353-3358

4. Sun YS, Zhao Z, Yang ZN, Xu F, Lu HJ, Zhu ZY, Shi W, Jiang J, Yao PP, Zhu HP (2017) Risk factors and preventions of breast cancer. Int J Biol Sci 13(11):1387

5. Caruso A, lacopetta D, Puoci F, Rita Cappello A, Saturnino C, Stefania SM (2016) Carbazole derivatives: a promising scenario for breast cancer treatment. Mini Rev Med Chem 16(8):630-643

6. Ng RC, Kassim NK, Yeap YS, Ee GC, Yazan SL, Musa KH (2018) Isolation of carbazole alkaloids and coumarins from Aegle marmelos and 
Murraya koenigii and their antioxidant properties. Sains Malaysiana 47(8):1749-1756

7. Huang L, Zhe-Ling FE, Yi-Tao WA, Li-Gen LI (2017) Anticancer carbazole alkaloids and coumarins from Clausena plants: a review. Chin J Nat Med 15(12):881-888

8. Zhou H, Li H, Cao Y, Sang X, Liu X (2019) Murrayanine exerts antiproliferative effects on human oral cancer cells through inhibition of AKT/mTOR and Raf/MEK/ERK signalling pathways In vitro and inhibits tumor growth in vivo. J BUON Off J Balk Union Oncol. 24:2423-2428

9. Mahapatra DK, Das D, Shivhare RS (2017) Substituted thiazole linked murrayanine-Schiff's base derivatives as potential anti-breast cancer candidates: Future EGFR Kinase inhibitors. Int J Pharm Sci Drug Res 9(3):139-144

10. Mahapatra DK, Shivhare RS, Kumar P (2018) Tert-butyl group bearing murrayanine-chalcone produced noteworthy anti-proliferative activity against breast cancer cell line. J Adv Res Biochem Pharmacol 1(3 \& 4):4-6

11. Zhang J, Gao Y, Ma C, Wang Y (2019) Murrayanine induces cell cycle arrest, oxidative stress, and inhibition of phosphorylated p38 expression in A549 lung adenocarcinoma cells. Med Sci Monitor 25:2002

12. Maleki P, Sadeghi Z, Shahryar Rahpeyma S, Taheri M, Raheb J (2020) MTT assay dataset of Polyethylenimine coated Graphenoxide nanosheets on breast cancer cell lines (MCF7, MDA-MB-231, MDA-MB-468). Hum Antibodies. 6:1-7

13. Sulthana S, Banerjee T, Kallu J, Vuppala SR, Heckert B, Naz S, Shelby T, Yambem O, Santra S (2017) Combination therapy of NSCLC using Hsp90 inhibitor and doxorubicin carrying functional nanoceria. Mol Pharm 14(3):875-884

14. Negarandeh $R$, Salehifar $E$, Saghafi $F$, Jalali $H$, Janbabaei $G$, Abdhaghighi MJ, Nosrati A (2020) Evaluation of adverse effects of chemotherapy regimens of 5-fluoropyrimidines derivatives and their association with DPYD polymorphisms in colorectal cancer patients. BMC Cancer 20(1):1-7
15. Lin SR, Chang CH, Hsu CF, Tsai MJ, Cheng H, Leong MK, Sung PJ, Chen JC, Weng CF (2020) Natural compounds as potential adjuvants to cancer therapy: preclinical evidence. Br J Pharmacol 177(6):1409-1423

16. Sun XY, Ma J, Li CJ, Zang YD, Huang JW, Wang XY, Chen NH, Chen XG, Zhang DM (2020) Carbazole alkaloids with bioactivities from the stems of Clausena lansium. Phytochem Lett 1(38):28-32

17. Satyavarapu EM, Sinha PK, Mandal C (2020) Preclinical development of mahanine-enriched fraction from indian spice Murraya koenigii for the Management of Cancer: Efficacy, Temperature/pH stability, Pharmacokinetics, Acute and Chronic Toxicity (14-180 Days) Studies. Biomed Res Int 10:2020

18. Renema N, Navet B, Heymann MF, Lezot F, Heymann D (2016) RANKRANKL signalling in cancer. Biosci Rep 36(4):e00366

19. van Dam PA, Verhoeven $Y$, Trinh XB, Wouters $A$, Lardon F, Prenen H, Smits E, Baldewijns M, Lammens M (2019) RANK/RANKL signaling inhibition may improve the effectiveness of checkpoint blockade in cancer treatment. Crit Rev Oncol Hematol 1(133):85-91

20. von dem Knesebeck A, Felsberg J, Waha A, Hartmann W, Scheffler B, Glas M, Hammes J, Mikeska T, Yan PS, Endl E, Simon M (2012) RANK (TNFRSF11A) is epigenetically inactivated and induces apoptosis in gliomas. Neoplasia 14(6):526

21. Sisay M, Mengistu G, Edessa D (2017) The RANK/RANKL/OPG system in tumorigenesis and metastasis of cancer stem cell: potential targets for anticancer therapy. OncoTargets Ther 10:3801

\section{Publisher's Note}

Springer Nature remains neutral with regard to jurisdictional claims in published maps and institutional affiliations.

\section{Submit your manuscript to a SpringerOpen ${ }^{\circ}$ journal and benefit from:}

- Convenient online submission

- Rigorous peer review

- Open access: articles freely available online

- High visibility within the field

- Retaining the copyright to your article

Submit your next manuscript at $\boldsymbol{\nabla}$ springeropen.com 